\title{
Long Term Consequences of the Fontan Procedure and How to Manage Them
}

Authors:

W. Aaron Kay, MD'

Tabitha Moe, $\mathrm{MD}^{2}$

Blair Suter, MD ${ }^{3}$

Andrea Tennancour, NP ${ }^{1}$

Alice Chan, NP

Richard A. Krasuski, MD ${ }^{5}$

Ali N. Zaidi, MD

1. Indiana University School of Medicine, Krannert Institute of Cardiology, IN

2. University of Arizona School of Medicine, Phoenix, AZ

3. Indiana University School of Medicine, Departments of Medicine and Pediatrics, IN

4. Children's Hospital at Montefiore, Montefiore Medical Center, Albert Einstein College of Medicine, NY

5. Duke University Health System, Durham, NC

*Address reprint requests to: W. Aaron Kay, MD. 1801 N. Senate Blvd Suite 2000, Indianapolis IN. Email: wkay@iuhealth.org

Emails of additional authors:

Moe TG:Tmoe@azcard.com

Suter BC: bcsuter@iupui.edu

Tennancour AE: atennancour@iuhealth.org

Chan A: ac3262@columbia.edu

Krasuski RA: richard.krasuski@duke.edu

Zaidi AN: azaidi@montefiore.org

\section{Disclosures:}

W. Aaron Kay: Nothing to disclose

Tabitha Moe: Nothing to disclose

Blair Suter, MD: Nothing to disclose

Andrea Tennancour, NP: Nothing to disclose

Alice Chan, NP: Nothing to disclose

Ali N. Zaidi, MD: Nothing to disclose

Richard A. Krasuski, MD: Dr. Krasuski serves a consultant and receives research funding from

Actelion Pharmaceuticals. He also serves as an investigator for Edwards Lifesciences and is an unpaid member of the scientific advisory board for Ventripoint.

This is the author's manuscript of the article published in final edited form as:

Kay, W. A., Moe, T., Suter, B., Tennancour, A., Chan, A., Krasuski, R. A., \& Zaidi, A. N. (2018). Long Term Consequences of the Fontan Procedure and How to Manage Them. Progress in Cardiovascular Diseases. https://doi.org/10.1016/j.pcad.2018.09.005 


\section{Abbreviations:}

A1AT = alpha-1-antitrypsin

$\mathrm{ACHD}=$ adult congenital heart disease

$\mathrm{AF}=$ atrial fibrillation

$\mathrm{AP}=$ atriopulmonary

ANZFR = Australia New Zealand Fontan Registry

ARNIs = Combined angiotensin-receptor and neprilysin inhibitors

$\mathrm{AP}=$ atriopulmonary Fontan

$\mathrm{AV}=$ atrioventricular

BDCPA = bidirectional cavopulmonary anastomosis

$\mathrm{BNP}=$ brain natriuretic peptide

$\mathrm{CO}=$ cardiac output

$\mathrm{CT}=$ computed tomography

CVP = central venous pressure

$\mathrm{EC}=$ extracardiac Fontan

$E F=$ ejection Fraction

GFR = glomerular filtration rate

$\mathrm{Gl}=$ gastrointestinal

$\mathrm{HF}=$ heart failure

HLHS = hypoplastic left heart syndrome

IART = interatrial reentry tachycardia

INR = international normalized ratio

IVC = inferior vena cava

VSD = ventricular septal defect

$\mathrm{LT}=$ lateral tunnel Fontan

MCS = mechanical circulatory support

$\mathrm{NOAC}=$ novel oral anticoagulant

NT-proBNP $=\mathrm{N}$-terminal pro-brain natriuretic peptide

$\mathrm{OHT}=$ orthotopic heart transplantation

$\mathrm{PA}=$ pulmonary artery

PDE5 = phosphodiesterase -5

PLE $=$ protein losing enteropathy

$\mathrm{PVR}=$ pulmonary vascular resistance

$\mathrm{RA}=$ right atrium

$\mathrm{SV}=$ single ventricle

SVR = systemic vascular resistance

TA = Tricuspid atresia

TCPC = total cavopulmonary connection

$\mathrm{TE}=$ thromboembolic

VKA $=$ vitamin $\mathrm{K}$ antagonists

$\mathrm{WHO}=$ World Health Organization 


\section{ABSTRACT}

In 1971, Fontan and Baudet described a surgical technique for successful palliation of patients with tricuspid atresia. Subsequently, this technique has been applied to treat most forms of functional single ventricles and has become the current standard of care for long-term palliation of all patients with single ventricle congenital heart disease. Since 1971, the Fontan procedure has undergone several variations. These patients require lifelong management including a thorough knowledge of their anatomic substrate, hemodynamic status, management of rhythm and ventricular function along with multi organ evaluation. As these patients enter middle age, there is increasing awareness regarding the long-term complications and mortality. This review highlights the long-term outcomes of the Fontan procedure and management of late sequelae.

Key Words: Adult congenital heart disease; Fontan 


\section{INTRODUCTION}

The Fontan procedure, devised by Fontan and Baudet was first performed in France well over 40 years ago [1]. Prior to the Fontan procedure, single ventricle (SV) patients rarely survived past early adulthood, and those that did were nearly universally cyanotic during their short lives. Theoretically, the Fontan operation separates the systemic and pulmonary venous returns to ameliorate the disadvantages of long-term hypoxemia, reduce thromboembolic (TE) events, preserve ventricular function, and prolong survival [2]. Candidates for the Fontan procedure must be carefully selected to give each procedure an optimal chance of succeeding. The ideal age for the Fontan operation is still debated despite advances in surgical techniques over the years.

Over time, the Fontan procedure has continued to evolve, leading to improved early and intermediate prognoses. However, it remains a palliative procedure and limited in its ability to fully eliminate the problems associated with SV physiology. Surgical approaches to palliating SV physiology must focus on ameliorating the two major challenges of this parallel circuit of blood flow: (1) cyanosis induced by mixing of systemic deoxygenated and pulmonary oxygenated blood and (2) chronic volume overload assumed by the single functional ventricle, which leads to dilatation, hypertrophy, and failure without palliation [3]. As Fontan patients move to adulthood, there remains a steady attrition rate attributable to several known complications of the procedure, with the three most common modes of death being heart failure (HF) from ventricular dysfunction, thromboembolism and sudden death [3, 4]. There remain several other long-term complications including residual cyanosis, arrhythmias, protein losing enteropathy (PLE) and Fontan associated liver disease (FALD) [5-11] 


\section{STAGED PALLIATIVE SURGERY AND FONTAN VARIATIONS}

The Fontan operation was first used in 1968 for the repair of tricuspid atresia (TA) [1]. It included the insertion of an aortic or pulmonary homograft valve, at both the inflow and outflow of the right atrium (RA). In 1973, Kreutzer et al described the use of the pulmonary valve of a patient with TA to connect the RA to the pulmonary artery (PA) [12] Since then, several different types of Fontan modifications have been devised (FIGURE 1). Management strategies in the neonatal period are directed toward providing an adequate source of pulmonary blood flow either by creating an aorticopulmonary shunt (such as a Blalock-Taussig shunt) to augment pulmonary blood flow or by restricting excessive pulmonary blood flow to ensure adequate systemic blood flow. An atrial septectomy or septostomy is done in most cases to ensure adequate pulmonary venous return to the systemic ventricle. The first cavopulmonary connection is created in the first year of life via a bidirectional Glenn shunt. The Fontan circulation is completed by connection of the inferior vena cava (IVC) to the PA once growth of the pulmonary vasculature is appropriate, usually between 1 and 5 years of age. This staged surgical process serves to slowly acclimate the pulmonary vessels to the increased blood flow and the SV to decreased volume load in a stepwise fashion $[3,13]$

Years ago, the Fontan connection was made by anastomosing the RA to the PA, hoping to maintain some pump function in the pulmonary circuit using intrinsic contractility of the RA. This atriopulmonary (AP) Fontan has since been replaced by the total cavopulmonary connections (TCPC), which have less kinetic energy loss and more streamlined blood flow. TCPC include lateral tunnel (LT) and the extracardiac (EC) conduits, which have improved survival and hemodynamic superiority over the AP technique $[3,14]$. The LT operation was introduced in the 1980s and creates a path from the IVC to the PA using a prosthetic baffle sutured to a portion of the lateral wall of the RA $[3,15]$. The EC conduit was introduced in the early 1990 s and uses a tube of synthetic graft material to connect the IVC to the PA, bypassing the RA entirely and 
minimizing risk of inducing atrial arrhythmias $[3,16]$. Other less common types of Fontan such as Bjork-Kreutzer modification and intracardiac Fontan have also been performed less often but are beyond the scope of this paper.

As TCPC operations have evolved over time, the AP Fontan has been abandoned due to excessive risk of arrhythmia, thrombosis, and early death. Many patients with AP Fontans have been converted to TCPC Fontans, although the indications for doing so are controversial and surgical experience and survival rates vary from center to center (FIGURES 2, 3) [7]. Lateral tunnel Fontan is preferred at some centers given ability to implant an adequate sized conduit in younger children ( $<24$ months of age), and improved ability to fenestrate versus an extracardiac Fontan [17]. However, at some centers, there has been a higher risk of sinus node dysfunction with LT Fontan versus EC Fontan. EC Fontan is also easier to implement in patients with complex venous anatomy, such as heterotaxy.[17] Since an EC conduit is unable to grow with the patient, in order to place an adequate-sized conduit that will last through adulthood, an EC conduit is typically placed at a later age than a LT [17].

Frequently, in high-risk patients, a small fenestration is created between the conduit and the pulmonary atrium to allow a residual right-to-left shunt, thereby limiting caval pressure and congestion, but increasing the preload of the systemic ventricle and cardiac output (CO) at the expense of mild cyanosis. Fenestrations may be electively closed later via percutaneous catheter techniques. Low-risk Fontan patients can be managed surgically without a fenestration, thereby eliminating the future need for closure $[3,18]$.

\section{LONG TERM SURVIVAL FOLLOWING FONTAN REPAIR}


As surgical techniques and management of patients after the Fontan operation have improved over the last 40 years, there has been improvement in early and intermediate-term survival [2]. However overall survival remains inferior compared to the general population, with gradual attrition due to long term complications including TE complications, HF, arrhythmias and sudden death [4]. To date, the largest series analyzed are at Mayo Clinic $(n=1052)$ [2] and the Australia/New Zealand Fontan registry (ANZFR) $(n=1089)$ [19]. A recently published, large meta-analysis reviewing long-term outcomes of 5859 patients showed AP Fontans had much worse survival than EC or LT types, though ventricular morphology did not impact survival [20].

The Mayo Clinic series reported 10-, 20-, and 30- year transplant-free survival rates of 74\%, $61 \%$, and $43 \%$, respectively [2]. These poor survival rates were likely due to a large proportion of the now-abandoned AP-type Fontans as well as poor survival due to the learning curve of the pioneering surgical era. Downing et al. presented long-term follow up of 773 consecutive patients who underwent a first Fontan operation at a single institution during an 18-year period. The 20 -year estimate for survival with intact Fontan circulation was $74 \%$. Independent risk factors for death or loss of Fontan circulation were identified, but several of these characteristics conferred risk only in the early post-Fontan period. Late outcome was predicted only by presence of AV valve regurgitation pre-Fontan and length of post-Fontan ICU stay. Ventricular morphology and type of Fontan operation were not associated with early or late outcome [21]. More recently, Zannino and colleagues described long term outcomes for 683 adult survivors of the Fontan procedure in ANZFR. Fontan type was AP connection in 201 and TCPC in 482 patients (249 LT and 233 EC). Survival for the entire cohort was $90 \%$ at 30 years and $80 \%$ at 40 years of age, with survival at 30 years of age significantly worse for AP patients [22]. The authors concluded that the long-term survival is improving, but significant morbidity and mortality remain for these patients. Of note, most patients (65\%) had systemic left ventricular 
morphology. Additional risk factors for poor prognosis included male gender and moderate or severe AV valve regurgitation [22].

It should be highlighted that the management of hypoplastic left heart syndrome (HLHS) has changed substantially over the past four decades. In the 1970s, children with HLHS could only be provided with supportive care and died within the neonatal period. The advent of the Norwood procedure in the early 1980s has greatly improved prognosis, and the majority of patients now undergo a series of three surgical stages that support survival into early adulthood [23]. Despite these advancements, patients with HLHS have considerable long-term morbidity following Fontan operation, and early mortality remains common [4, 23]. Pundi et al. reported that only $40 \%$ of children with HLHS survived all three surgical stages and remained alive 10 years after Fontan completion [2]. High systemic venous pressures, abnormal ventricular morphology, TE events, and recurrent arrhythmias create a tremendous burden of disease for these children as they reach adulthood. Exercise intolerance, HF, hepatic dysfunction, and disease processes more specific to patients with Fontan physiology such as PLE and plastic bronchitis are additional complications that can occur and further negatively impact the quality of life of patients with HLHS [24].

Survival for patients with HLHS who undergo Fontan completion ranges from $72 \%$ to $85 \%$ at 10 years $[4,25]$. However, when accounting for all the surgeries required from infancy, the overall survival drops to only $50 \%$ to $70 \%$ at 10 years $[25,26]$. Despite this, there are now a small number of HLHS survivors reaching adulthood [27]. In a recent multicenter study of 543 patients committed to Norwood surgery who could have reached 18 years of age by 2014 , transplantfree survival to adulthood was only $14 \%$, with most mortality driven by the perioperative period of the Norwood procedure (41\%). As post-Norwood and interstage mortality continues to decrease, it is expected that the number of adult HLHS patients will continue to grow [28]. 


\section{LONG TERM COMPLICATIONS FOLLOWING FONTAN REPAIR}

\section{Heart Failure}

$\mathrm{HF}$ after Fontan is very different from and much more complex than $\mathrm{HF}$ in acquired heart disease. Fontan failure is a clinical syndrome in which circulation can no longer meet the metabolic demands of the body. Patients with Fontan failure predominately suffer from systemic venous congestion. Four phenotypes of Fontan failure have been established with renal hypoperfusion as the common terminal pathway for all phenotypes [29].

(I) Fontan failure with reduced ejection fraction (EF), which most closely resembles acquired systolic HF, is characterized by signs and symptoms of low EF and is the most common type of HF seen in the pediatric Fontan population.

(II) Fontan failure with preserved EF, which is predominated by pulmonary venous congestion, elevation of Fontan pressures and hepatic congestion.

(III) Fontan failure with normal pressures, which has a clinical presentation of right-sided HF including congestion, hepatomegaly, splenomegaly, ascites, varicose veins, and exercise intolerance despite normal EF and hemodynamics. Type III is caused by multi-organ system dysfunction, including portal venous outflow obstruction and adverse pulmonary vascular remodeling, making this group of patients particularly challenging to treat.

(IV) Fontan failure with abnormal lymphatics, which can occur in the setting of normal ventricular function and normal hemodynamics; this presents with plastic bronchitis (more common in children) and PLE.

A catheterization-based analysis by Hebson et al. described a phenotype of HF in the adult Fontan population that is hemodynamically distinct from traditional HF and is characterized by low systemic vascular resistance (SVR) and preserved CO [30]. In this study, the adult Fontan 
population had higher central venous and pulmonary capillary wedge pressures than the pediatric Fontan cohort. However, SVR was lower in the adults and CO was preserved, even in patients with more severe symptoms. In a subanalysis comparing failing Fontan physiology in children versus adults with a systemic left ventricle, no significant difference in systolic ventricular function was seen. However, adults with a systemic right ventricle had a significantly higher incidence of systolic ventricular dysfunction than children.

Adult Fontan patients with Fontan failure more closely resemble the hemodynamic profile of portal hypertension (phenotypes II and III as above), with pathology secondary to time-related exposure to elevated post-sinusoidal Fontan pressure, leading to liver damage and eventually portal hypertensive-type circulatory derangement. Of note, failing Fontan patients with preserved systolic function appear to do worse after orthotopic heart transplantation (OHT) than patients with decreased systolic function [31]. This paradoxical finding may be due to the limited ability to augment $\mathrm{CO}$ above a certain threshold from inability to augment stroke volume, regardless of EF, given lack of a venous capacitance chamber. [32-35]. Studies have demonstrated that skeletal muscle and ventilator pumps account almost entirely for the modest increase in stroke volume during exercise in patients with Fontan circulation. Fontan patients have little hemodynamic reserve to overcome a decrease in venous return, and are essentially dependent on heart rate to augment $\mathrm{CO}$ [36].

Since the phenotype of Fontan failure with preserved systolic function is distinct from that of the traditionally-accepted HF model, it brings into question whether treatment should resemble currently accepted HF therapy or rather the treatment for portal hypertension. Medications traditionally used in HF such as systemic vasodilators may not be well tolerated, and an approach more specifically tailored to the unique hemodynamics of the Failing Fontan may be 
more beneficial [30]. It is also unclear how traditional diagnostics such as biomarkers and imaging aid in diagnostics and evaluation of the Failing Fontan patient.

Brain natriuretic peptide (BNP), N-terminal pro-B natriuretic peptide (NT-proBNP), and serum sodium (hyponatremia) are biochemical parameters often monitored for HF exacerbations and have been shown to predict outcome in traditional HF. Use of biomarkers in Fontan patients continues to be investigated. Baggen et al. showed that elevated NT-proBNP levels were associated with increased risk of death or HF exacerbation [37]. BNP elevation has been shown to be a predictor of Fontan failure, mortality, and morbidity in adult patients in multiple studies $[38,39]$. Hyponatremia has also been shown to be very common in adults with congenital heart disease (ACHD) and carries a high risk of mortality [40]

In the Failing Fontan patient, cardiac and non-cardiac fibrosis are common and precede adverse outcomes in adulthood; therefore, biomarkers assessing fibrosis may be useful in assessing risk. These include galectin-3, a carbohydrate-binding protein, which is associated with a variety of biological effects including inflammation and fibrosis. Opotowsky et al. showed that galactin-3 levels were elevated in Fontan patients compared with a control group, and correlated with worsened kidney function, advanced age, and higher risk of hospital admission or death. Galectin-3 levels did not correlate with specific underlying ventricular morphology nor type of Fontan procedure [41].

The paradigm of traditional HF treatment has two distinct scopes: symptomatic relief and mortality benefit. Symptomatic therapies focus both on traditional HF symptoms as well as unique Fontan-related problems such as plastic bronchitis, protein losing enteropathy (PLE), and recurrent ascites. Given the lack of randomized controlled trials of HF therapy in Fontan patients, use of traditional HF medications is still widespread, with only very small trials and 
expert opinion to guide decision-making. In 2016, a systematic review of drug therapy trials in Fontan patients revealed only nine small trials with a total of 267 Fontan patients studied.

Sildenafil was the drug most commonly examined [42]. Current evidence does not support the routine use of ACE inhibitors, $\beta$ blockers, or pulmonary vasodilators in this population [43] and the lack of data has led to difficulty in treatment standardization.

In a large Fontan registry, ACE inhibitors were used in about one third of Fontan patients and were almost twice as likely to be used in patients with HLHS than alternative morphologies. Only one third of patients given ACE inhibitor had an indication that would justify its use in a two-ventricle circulation [44]. In this study, indications for ACE inhibition included ventricular dysfunction (either systolic or diastolic), AV valve regurgitation, expectation that an ACE inhibitor would preserve ventricular function, history of prolonged effusions during initial Fontan surgery, and systemic hypertension [44]. Since failing Fontan physiology is often similar to hepatorenal syndrome, there is some concern that ACE inhibitors might be ineffective and potentially harmful in patients with type III Fontan Failure [45]. Additionally, ACE inhibitors have shown inability to improve exercise capacity or hemodynamics in pediatric and adolescent patients in at least one study [46].

$\beta$-blockers have shown mixed results in both pediatric and adult Fontan populations. It is wellknown that Fontan patients are dependent on heart rate to raise $\mathrm{CO}$ given the inability to significantly augment stroke volume with just one ventricle [36], thus lowering heart rate too much may have deleterious effects on $\mathrm{CO}$. On the other hand, a lower heart rate may improve diastolic filling, especially in patients with atrial arrhythmias, which might improve CO. In a randomized controlled trial by Shaddy et al., $\beta$-blocker therapy did not improve outcomes for pediatric and adolescent Fontan patients with CHF [47]. Other studies have shown more promising results with $\beta$-blockers, including improvement of functional classification and 
symptoms, [48-50] right ventricular remodeling, and improved exercise duration [51]. Combined angiotensin-receptor and neprilysin inhibitors (ARNIs), which have been extensively studied in acquired HF[52] could have benefit for Fontan patients with reduced EF and deserve further study. Pulmonary vasodilators may increase cardiac filling in Fontan patients via lowering pulmonary vascular resistance (PVR), but have not consistently shown durable clinical outcomes. Sildenafil has been shown to improve echocardiographic indices of myocardial performance [33]. Bosentan has been shown to have variable outcomes on exercise capacity and functional class in Fontan patients $[53,54]$. In a small, randomized-controlled trial by Cedars et al., ambrisentan had a statistically significant, but clinically modest, improvement in exercise capacity [55]. One case report also demonstrated improvement in exercise capacity after treatment with macitentan [56].

Progress with mechanical circulatory support (MCS) in SV patients has been limited, but remains increasingly important with growing populations of sick Fontan patients and limited supply of donor organs. The exact number of Fontan patients requiring transplant in the United States is elusive, as the United Network of Organ Sharing does not publish specific diagnoses, but rather lumps all congenital heart disease into a single group, including SV patients along with patients with two-ventricle physiology. Most studies have focused on supporting patients with a failing systemic ventricle with limited reports of ventricular assist device support of the pulmonary circulation [57]. Available ventricular assist devices provide flows that are too high for the pulmonary vascular bed, and new devices are being developed to overcome this issue. Rodefeld et al. are performing preclinical testing on a novel Fontan-specific cavopulmonary assist device, which has a goal of providing a modest pressure boost to existing blood flow to effectively restore a more stable biventricular status [58]

$\mathrm{OHT}$ in Fontan patients brings many distinct challenges and considerations. The transplant surgery itself involves significant effort to take down the Fontan to reconstruct connections for 
the transplanted heart, and all Fontan patients have had extensive prior surgery. The exact timing of $\mathrm{OHT}$ evaluation in Fontan patients remains uncertain. Determining if enough decline has occurred to justify $\mathrm{OHT}$, while at the same time planning for OHT early enough so a patient will survive and therefore benefit is an important but difficult process. Criteria to help determine timing for transplant include a combination of declining functional status and changes on imaging, biomarkers, and cardiopulmonary exercise testing [59]. In the United States, only a very small minority (3.3\%) of adult $\mathrm{OHT}$ occur in $\mathrm{CHD}$ patients [60]. Indications for $\mathrm{OHT}$ after Fontan include systemic ventricular failure refractory to medical therapy, chronic passive congestion, intractable ascites, and progressive cyanosis leading to functional decline. Currently, OHT wait-list mortality or delisting due to worsening clinical status is disproportionately common for ACHD patients listed as status 1A in the United States [61]. It is controversial if the current allocation system is appropriate for ACHD and whether it should be adapted given the unique aspects of this patient population [62]. Given the high incidence of FALD, some centers have started performing combined heart-liver transplants, although there overall is very limited experience with this technique; and very few centers have performed more than 10 combined OHT-liver transplants on Fontan patients [63].

\section{Thrombosis}

Despite changes in the surgical design of Fontan, and although the AP Fontan has largely been abandoned, thrombosis continues to be a major problem following the Fontan procedure [64]. The reported prevalence of thromboembolic (TE) events is as high as $33 \%$, contributing to approximately $25 \%$ of mortalities via deep venous thrombosis, pulmonary embolism, or cerebrovascular accident [64-66]. Thrombosis risk factors include non-pulsatile pulmonary flow, low cardiac output, arrhythmias, type of Fontan connection, residual shunts or intentional fenestration, dilated atrium, ventricular dysfunction, hypoxia, liver disease, PLE, and underlying coagulation abnormalities [65-67]. In some small studies, Fontan patients have been found to 
have decreased levels of anticoagulant proteins (proteins $\mathrm{C}$ and $\mathrm{S}$ ) and an increase in prothrombotic factors (factor VIII and von Willebrand factor). [65].

Most clinicians treating Fontan patients agree with routine use of pharmacologic prophylaxis against TE events, but there is no consensus on what specific medication(s) should be used $[65,67]$. Some authors favoring antiplatelet medications (usually aspirin), while others favor vitamin $\mathrm{K}$ antagonists (VKA) such as warfarin. In a large meta-analysis on thromboprophylaxis in Fontan patients, aspirin was statistically noninferior to VKA with regard to TE events, but patients in neither group had many TE events [65]. A retrospective multicenter study (TACTIC) evaluating anticoagulation therapy for atrial arrhythmias (in a wide variety of CHD types, including Fontan patients) also showed no significant difference between the use of antiplatelet or VKA therapy in the prevention of TE events, though bleeding events were higher in the VKA group ( $\mathrm{p}=0.039)$. Of note, patients with more complex congenital heart lesions were more likely to have a TE event $(p<0.001)$, and risk scores used in general adult cardiology to predict TE events $\left(\mathrm{CHADS}_{2}\right.$ and $\mathrm{CHA}_{2} \mathrm{DS}_{2}$-VASc scores) did not accurately predict TE events in Fontan patients [68]. Events in VKA patients often are caused by failure to maintain a therapeutic International Normalized Ratio (INR); and some studies have suggested that well-managed pharmacist-assisted protocols can help reduce overall event rates of both bleeding and thrombosis [69].

Given the intrinsic limitations of VKA therapy, novel oral anticoagulant (NOAC) therapy to prevent TE events is increasingly being considered in Fontan patients, despite very limited data regarding safety or efficacy [70]. NOACs are often favored as they do not require blood draws for INR monitoring, have no dietary interactions, and may lead to better medication compliance and improved quality of life [71]. The NOTE registry has been actively recruiting patients to determine effectiveness and safety of NOACs in ACHD patients. A recent publication from 
NOTE showed only 8 minor events in the first 30 days after starting a NOAC for arrhythmia in 99 patients. Events included nonfatal bleeding and other minor side effects in patients who transitioned from VKA to NOAC therapy, and there were no reported adverse events in those who were started on a NOAC without prior anticoagulation [71]. Another study evaluating NOAC use in Fontan patients resulted in only one thrombotic event and no major bleeding, though this was a small $(n=21)$, retrospective, single-center study [72].

The use of anticoagulation in patients with the Fontan procedure to prevent TE events has been associated with fewer hospitalizations and a lower risk of death [67]. Being on either aspirin or VKA therapy is clearly superior to no such therapy for Fontan patients. At this time, the agent(s) used is generally based on regional institutional preferences and further data is awaited [73].

\section{Arrhythmias}

Arrhythmias, particularly atrial tachyarrhythmias, are extremely common in the Fontan population. Due to proximity of the surgical Fontan connections to the sinus node, iatrogenic sick sinus syndrome is quite common. Catheter ablation as well as pacemaker placement are much more challenging in Fontan patients than in patients with two-ventricle circulations.

In congenital heart disease populations, atrial fibrillation (AF) incidence increases with aging, higher complexity of ACHD, and increased numbers of surgical interventions (due to resulting surgical scar). Acquired cardiovascular comorbidities, such as hypertension, obesity, sleep apnea, and coronary disease, appear to increase the incidence and burden of AF; and as Fontan survivors are aging, these acquired cardiovascular conditions are becoming more prevalent. Atypical atrial flutter and incisional interatrial reentrant atrial tachycardia (IART) are exceptionally common due to direct injury and scarring of the right atrium from the initial surgery, as well as due to post-Fontan alterations of progressive right atrial dilation and 
hypertrophy [74]. AP Fontan procedures may lead to right atrial gigantism, thus compressing the right upper pulmonary vein and leading to AF. Approximately $50 \%$ of patients with right atrialright ventricular (Bjork) or AP Fontan procedures develop atrial tachycardia within 10 years of their palliative procedure. Improved surgical techniques have led to a diminishing burden of atrial arrhythmias, but they remain very common [75].

Patients with residual cyanosis after Fontan are also at increased risk for AF due to chronic subendocardial ischemia given limited oxygen reserves and high viscosity [76]. Generally, ACHD patients tolerate AF more poorly than the general population and have a higher risk of TE events and hemodynamically instability at rapid heart rates. Also, Fontan patients tend to develop AF at a much younger age than the general population, and AF is more challenging to manage with medications or catheter-based and/or surgical ablation procedures.

Amiodarone has been used for adults since the 1960 s and is widely considered to be the most effective antiarrhythmic drug, including in the ACHD population. It should be administered with caution in the latter group because of its daunting long-term side-effect profile, which includes potential harm to the thyroid, lungs, liver, and eyes [77]. Fontan patients with arrhythmias are more like to be refractory to single-drug antiarrhythmic therapy, have depressed myocardial function, and be intolerant of drugs with negative inotropic effects [78]. Patients with double inlet left ventricle who underwent surgical repair before the routine use of the Damus-Kaye-Stansel reconstruction have a pulmonary artery stump that is oversewn with a resultant pulsatile swirling of blood, creating a high-risk nidus for thrombus formation. This type of repair necessitates lifelong anticoagulation, even in the setting of normal sinus rhythm [79].

Although the incidence of atrial tachycardia after Fontan surgery is high, access to the pulmonary venous atrium, a frequent site of arrhythmia origin, is often difficult and potentially 
risky, as it requires a transbaffle puncture in a TCPC Fontan (unless there is a patent fenestration). In AP Fontan patients, the right atrium remains connected to the great veins, but getting across the septum is very difficult due to the degree of atrial enlargement and hypertrophy as well as the lack of commercially available trans-septal catheters long enough to reach target areas.

When traditional percutaneous access is difficult or impossible, other routes can be considered. Nehgme et al. descripted utilizing direct puncture of the chest wall (percutaneous transthoracic access) with fluoroscopic guidance to obtain access to the pulmonary venous atrium in six LT Fontan patients [80]. Mapping and ablation catheters were placed in the pulmonary venous atrium, and additional electrophysiologic catheters were placed within the Fontan baffle and esophagus. Complications included one pneumothorax and two hemothoraces requiring drainage. Another new procedure that was recently described involves CT-guided transconduit puncture in patients with EC Fontan, in which the dilator tip is grasped with a snare catheter [81]. Subxiphoid and epicardial approaches may be used in patients without ACHD, however, they are generally not employed for patients with ACHD. Both these approaches rely on the ability to access the pericardial sac, which in Fontan patients is likely to be immobile and scarred with thick, fibrous adhesions [82].

Surgical ablation is considered the gold standard for invasive rhythm control in SV patients. Early surgeries involved conversion of AP Fontan to TCPC Fontan in conjunction with surgical ablation for AF [83]. Fontan conversion that did not include surgical ablation has been associated with a high incidence of postoperative atrial arrhythmias [84].The surgical approach has since progressed from isthmus ablation, to a modified right-sided maze procedure, and eventually to the MAZE-Cox III (right and left-sided maze) procedure for AF $[85,86]$. 
With three-dimensional mapping and pace-mapping techniques, the focus of ventricular arrhythmias can be marked by an ablation catheter. This allows for the localization and surgical excision of the arrhythmogenic tissue, thereby substantially reducing the possibility for future ventricular arrhythmias and resultant ICD discharges [87]. SV patients scheduled for Fontan revision may be appropriate candidates for similar hybrid approaches that include surgical resection of arrhythmogenic atrial tissue.

With advanced planning, patients undergoing open-thoracotomy procedures can have epicardial leads placed intraoperatively, potentially avoiding another open sternotomy or thoracotomy if they develop a future pacing indication. Therefore, surgical ablation should always be discussed whenever an open heart surgical procedure is planned in these patients. Finally, elective surgical excision of thrombogenic structures such as the left atrial appendage and residual, ligated, blind-ended pulmonary artery stump should be performed if feasible and with low enough risk at the time of concomitant open heart cardiac surgical repairs.

\section{Residual Cyanosis}

Cyanosis late after Fontan is common. Fontan patients may remain mildly hypoxemic, even after full repair and closure of atrial level shunts. Cyanosis can significantly worsen over time. Desaturation is secondary to multiple causes including an intentionally placed surgical fenestration, persistent coronary sinus drainage into the right atrium, pulmonary arteriovenous malformations, aorticopulmonary collaterals, or venovenous collaterals [3]. The degree of cyanosis may not be readily apparent at rest and can be unmasked by exercise. Persistently cyanotic Fontan patients without a known fenestration may benefit from interventional catheterization by an appropriately trained operator who can evaluate and treat these various causes of right-to-left shunts by deploying a variety of coils and devices (FIGURE 4). [70, 88]. Unfortunately, clinically significant venous collateral channels and other right-to-left shunts tend 
to recur after coiling or device closure [89]. Closure of venovenous collaterals in some series has been found to be associated with a higher overall mortality rate [90]. If cyanosis cannot be corrected (or is accepted in a patient with intentional fenestration), appropriate preventative measures to avoid air embolism should be instituted such as the use of air filters with intravenous lines. Anticoagulation therapy is recommended for all patients with cyanosis or fenestration post-Fontan due to the risk of paradoxical systemic TE [70].

\section{Protein Losing Enteropathy}

PLE is a rare complication that can develop in patients following Fontan palliation, occurring in $5-15 \%$ of patients $[91,92]$. PLE may present as early as a few weeks after the Fontan operation, but on average presents $3-8$ years after Fontan surgery [93, 94]. PLE is manifested by the severe loss of proteins, immunoglobulins and clotting factors via the gastrointestinal (GI) tract. A prevailing theory is that serum protein enters the lumen of the GI tract due to a loss of the intestinal barrier to protein permeability [95]. Typically, patients will have diarrhea due to excessive protein losses and subsequent bowel wall edema; however, not all patients present with diarrhea. Other symptoms may include abdominal cramping, ascites, electrolyte disturbances, peripheral edema, weight loss/gain, fatigue, and pleural or pericardial effusion [93]. Muscle tetany may occur secondary to hypocalcemia and thromboembolism is also a concern due to hypercoagulability. PLE can result in significant morbidity and mortality for this patient population, with a 5-year survival after diagnosis of only 46-59\% [96].

If PLE is suspected, diagnosis can be confirmed with testing of serum levels of albumin and protein, and testing for presence of elevated stool $\alpha$-1-antitrypsin (A1AT). A1AT is a protein that is normally excreted in only small amounts into the stool, thus elevated levels are an indicator of protein leak into the intestinal mucosa. A 24-hour stool A1AT clearance study is more sensitive than random stool levels of A1AT [94]. Unfortunately, no single test can definitively confirm or 
refute the diagnosis of PLE in the Fontan population. A proposed set of diagnostic criteria includes the presence of clinical manifestations of PLE, decreased serum albumin levels to $<3.5$ $\mathrm{g} / \mathrm{dl}$, serum protein $<6.0-6.3 \mathrm{~g} / \mathrm{L}$, and fecal A1 AT clearance $>27 \mathrm{ml} / 24 \mathrm{hr}$ without diarrhea and $>56 \mathrm{ml} / 24 \mathrm{~h}$ with diarrhea [97].

There are several hypotheses about what leads to the development of PLE in the Fontan population. Systemic venous pressure is chronically elevated in the Fontan circuit, leading to chronic passive venous congestion of the liver and intestines, which results in an increase in tissue and lymphatic fluid generation [94, 95]. The intestinal lymphatics become engorged and proteins are "lost" into the intestinal lumen. Intravascular oncotic pressure is reduced and fluid leaks out of the vascular spaces and into the interstitium, which results in peripheral edema, ascites and effusions that are common in patients with PLE [96]. PLE presents when the patient's ability to resynthesize lost protein is exceeded by the amount excreted in the intestine [94]. Chronically low CO in the Fontan circulation also results in poor perfusion of the Gl mucosa, which results in chronic inflammation, and an increase in interferon-y, tumor necrosis factor- $\alpha$, and serum albumin transmission across the intestinal mucosa. Elevated BNP levels can contribute to PLE by impeding lymph transport. Since not all patients with the Fontan circulation develop PLE, it has been hypothesized that individuals who develop PLE may have a congenitally abnormal lymphatic system [94].

Treatment for PLE is targeted towards decreasing protein loss and reducing symptoms by decreasing CVP, improving cardiac function, reducing inflammation, and improving nutrition [96, 98]. Identifying reversible hemodynamic abnormalities is an important step in treatment. Obstruction to systemic venous flow, which elevates venous pressures, should be corrected with balloon angioplasty and stenting [99]. Creating a Fontan fenestration (generally by catheterization) results in a Fontan pressure "pop-off," which can lower Fontan pressure and 
improve systemic cardiac output, although at the expense of reducing oxygen saturation. Fontan fenestration is rarely a long-term solution, however, given the high incidence of spontaneous fenestration closure, and thus it is generally considered to be a bridge to cardiac transplant or Fontan revision [100]. Atrial or ventricular pacing to increase heart rate may in some cases help improve symptoms via increasing CO [91].

Pharmacological treatments include loop diuretics and aldosterone antagonists to decrease edema. Afterload reducing medications such as ACE inhibitors and angiotensin receptor blockers may improve cardiac output by reducing SVR. Pulmonary vasodilators such as phosphodiesterase-5 (PDE5) inhibitors may help reduce PVR and subsequently CVP. PDE5 inhibitors have also been shown to improve mesenteric arterial blood flow. Corticosteroids can reduce inflammation; however long-term use leads to other well-known complications of long term steroid use. Oral budesonide is more enteric-specific with less systemic absorption, which limits systemic side effects; and therefore is often used for long term management of PLE [101]. Heparin has been shown to decrease inflammation (by inhibiting mast cell activity in the intestine), and also provides a barrier to larger molecules such as albumin [96]. Heparin can also reduce the occurrence of pulmonary and mesenteric micro-thrombi. Octreotide mimics natural somatostatin and can decrease thoracic duct lymphatic flow [93].

Diet modification is also an important component of treatment. Malnutrition and failure to thrive are common sequelae of PLE. As a result, attention to incorporating a high protein diet $(>/=2$ $\mathrm{g} / \mathrm{kg} / \mathrm{d}$ ), high medium chain triglycerides, low sodium, and low fat $(<25 \%$ calories) diet may also benefit patients [94].

Recent innovations have led to a focus on lymphatic system interventions for treatment of PLE. Liver lymphangiography is a useful tool in identifying dilated liver lymphatic ducts and can 
demonstrate leakage into the intestine [102-104]. The lymphangiography is performed in conjunction with endoscopy. Blue dye is injected into the lymphatic vessels, and leaks are identified via endoscopy. These vessels can then be embolized, and this has been shown to temporary normalize serum albumin blood levels and result in symptomatic improvement, though there are significant risks to the procedure and more studies are needed to assess longterm outcomes [104].

\section{Fontan-Associated Liver Disease}

FALD is a common complication of the Fontan circulation, which has been recognized with increasing frequency in adolescents and adults. All types of Fontan connection, including AP Fontan and the newer TCPC Fontan, are at risk for FALD [105]. The spectrum ranges from just mild histologic changes, to abnormal liver function tests, and to fulminant liver failure [106]. The etiology of FALD is not entirely known, but is likely multifactorial, with physiological derangements, particularly elevation in CVP, contributing to liver pathology $[8,107]$. Other likely contributing causes include chronic lack of tissue oxygenation, lack of pulsatility of pulmonary arterial flow, and other medical and surgical complications [107]. Risk factors for FALD include higher Fontan pressures, increasing age, longer duration of Fontan, underlying hepatitis B or C, alcohol use, and hepatotoxic drug use [8].

The best way to evaluate for FALD is uncertain, and there are no established predictors of which patients are at greatest risk for developing it. Patients after Fontan with frank cirrhosis have reduced life expectancy, even after heart transplant. It remains to be seen what effect on long-term survival milder forms of hepatic fibrosis have in Fontan patients [108].

The lack of reliable tests to determine severity and progression of FALD is a significant clinical problem. Several clinical, imaging, and laboratory elements have been identified in an effort to 
determine a useful scoring system to help risk-stratify these patients (such as the MELD and VAST scores) [109-112]. Basic serum liver function tests including aminotransferases, alkaline phosphatase, bilirubin, coagulation markers, and protein levels have generally not correlated with degree of liver disease [11]. Other evaluation for FALD includes liver imaging, cardiac MRI, liver biopsy, and transient liver elastography by ultrasound or MRI. Given the concerns of high venous pressure being the culprit for hepatic fibrosis, many studies have compared hemodynamic evaluation with liver findings. Noninvasive measurement of Fontan pressure, unfortunately, has been elusive; thus direct pressure measurement in the catheterization laboratory is generally considered when accurate hemodynamic assessment is needed. Several single-center studies have been done evaluating different modalities, but data sets are limited and generally do not include hemodynamic data, making it difficult to draw any conclusions.

Symptoms of hepatic dysfunction in Fontan patients can be very subtle, and synthetic function is often preserved despite severe hepatic involvement. Ascites is common, but esophageal varices are rarely found. Primary portal hypertension is rare in Fontan patients, and fibrosis is instead generally due to high systemic venous pressures [95]. Chronic CVP elevation (> 20 $\mathrm{mmHg}$ ) is known to greatly increase risk for hepatic fibrosis, but significant and possibly irreversible hepatic dysfunction may occur with chronic CVP in the mid-teens [95]. The chronicity of CVP elevation may be more important than the degree of CVP elevation. Rychik et al. demonstrated an eight-fold higher degree of hepatic collagen deposition in Fontan patients compared to non-Fontan controls and duration of time after Fontan completion correlated best with degree of fibrosis [95].

Abnormal liver histology is present in nearly all patients after Fontan surgery. These findings range from mild fibrosis to full cirrhotic changes. In a recent series of 74 consecutive young 
Fontan patients (mean age 16 years) undergoing liver biopsies, all biopsies had at least some degree of liver fibrosis, with some demonstrating advanced cirrhosis [113]. Wu et al. performed a retrospective analysis of liver histopathology samples available in 68 adult and pediatric Fontan patients, including both autopsy and biopsy specimens. Every specimen had centrilobular fibrosis, with $41.2 \%$ grading as severe. Portal fibrosis was found in $82.3 \%$ of specimens (14.7\% with cirrhosis) and centrilobular fibrosis grade was worse in patients with a dominant single ventricle compared to a combined right and left systemic ventricle [114]. Despite the high incidence of abnormal histopathology, neither centrilobular fibrosis nor portal fibrosis grade correlated with transplant-free survival or overall survival.

Although there is a high incidence of hepatic fibrosis in Fontan patients, liver biopsy findings have not correlated well with clinical outcomes. Liver biopsy results can be misleading, as fibrosis is patchy, and it is easy to obtain a false negative or false positive sample[115]. Standard pathologic grading systems may mischaracterize hepatic fibrosis patterns in Fontan patients, since the areas of fibrosis are different in Fontan patients than in non-Fontan patients with portal hypertension [95].

Prevention of FALD is an important but elusive clinical goal. This can be attempted by optimization of anatomy and physiology, as well as prevention of liver injury both prior to and after Fontan. Additional preventive strategies include immunization against and treatment of viral hepatitis, as well as avoidance of hepatotoxins and obesity (steatohepatitis). Moderation of alcohol intake should be considered, although the effects of alcohol on FALD are unknown [8].

Liver function biochemistries are often normal, even in the presence of significant liver fibrosis in Fontan patients. Given the concerns about potentially severe risks and uncertain benefits of liver biopsy, other testing has been developed. The MELD score (Model of End Stage Liver 
Disease) has commonly been used to risk-stratify patients in the general population to allocate organs for liver transplantation [112] as well as to risk-stratify cirrhotic patients who need openheart surgery [116]. This score is a mathematical model including serum creatinine, sodium, bilirubin and INR, as well as a correction variable if the patient is on dialysis. Scores range from 6 to 40 , with higher scores being associated with a higher risk of death due to severe liver disease.

Many Fontan patients are managed on chronic vitamin $\mathrm{K}$ antagonists, as their INRs are elevated out of proportion of the degree of liver disease; thus the MELD-XI score (MELD excluding INR) may have better prognostic value. MELD-XI correlates with degree of hepatic fibrosis [111], as well as mortality and need for heart transplant in adult Fontan patients [110, 117]. Laboratory data in these patients usually demonstrates only mild abnormalities, even when advanced cirrhosis is present [118], making assessment of liver dysfunction with tools such as the MELD score [119] of limited clinical value. Consequently, the search for a more meaningful measure of liver function in this population is critical and remains ongoing [11].

Elder et al. evaluated a novel risk score to determine risk of portal hypertension after Fontan they termed the VAST score (varices, ascites, splenomegaly, and thrombocytopenia)[109]. The score includes one point for each of these features, and a score $\geq 2$ points was considered to be consistent with portal hypertension. This score was then validated retrospectively against 73 adult and pediatric Fontan patients using logistic regression. Features of portal hypertension, as identified as a VAST score $\geq 2$ were found in $36 \%$ of the cohort; and VAST score $\geq 2$ after adjusting for other variables resulted in a 9-fold increased risk of adverse events.

Given the increasing morbidity associated with liver disease, identifying it early and with less invasive assessment has become paramount. Imaging has been shown to accurately identify 
structural liver disease, but unfortunately not the degree or severity [3, 120]. Abdominal ultrasound with Doppler evaluation should be performed at baseline with consideration for elastography. Transient elastography by ultrasound or MRI has been evaluated by several groups as a manner for assessing hepatic stiffness. Its role, however, remains limited by difficulty in distinguishing edema from fibrosis [121]. Cardiac MRI routinely includes the liver in its field of view, and thus serial changes of liver can be evaluated as part of routine follow-up MRI. In a recent study, Lewis et al measured IVC, right hepatic vein size, and spleen diameter in Fontan patients who had undergone serial cardiac MRI. Over a mean time of 5.1 years, a significant increase in hepatic dimensions, right hepatic vein diameter, and mean spleen diameter was seen [105]. In 55\% of patients, increasing hepatic congestion was found between studies. Progressive hepatic congestion correlated with reduced SV EF $<50 \%$, degree of ventricular dilation, and a higher MELD-XI score. No association was between biochemical abnormalities and change in anatomic variables. Interestingly, only right hepatic vein diameter correlated with time from Fontan completion[105].

Arguably the most devastating liver-related complication after Fontan palliation is the development of hepatocellular carcinoma (HCC) [122]. Precise incidence late after Fontan palliation is unknown. A recent nationwide study of all 2700 Fontan procedures in Japan demonstrated a prevalence of $1.15 \%$ [122]. Treatment for HCC can include resection, chemoembolization, or in advanced cases, liver transplant, which may need to be combined heart-liver transplant. Given advanced tumor burden at diagnosis in many cases, palliative care is often considered. Outcomes and risk factors for HCC are also unknown in the Fontan population. Diagnosis of HCC can be made by combining imaging, alpha fetoprotein levels, and biopsy $[123,124]$. Most reported cases of HCC have involved biopsy, although biopsy is unnecessary in cases with exceedingly high AFP levels. In some cases, nodules that satisfy classic criteria for HCC have been found to be benign on biopsy in Fontan patients [125]. 
Testing serum alpha-fetoprotein and performing liver imaging every six months should be considered in patients with established FALD due to the increased risk for hepatocellular carcinoma [8] .

There are no current medical therapies recommended for routine treatment of hepatic fibrosis in the Fontan population. The renin-angiotensin-aldosterone system is recognized as an important regulator of liver fibrosis, and there is evidence supporting anti-inflammatory and anti-fibrogenic effects of ACE inhibitors and angiotensin receptor blockers in the general population with liver disease [126]. However, no similar studies have been performed in FALD. Caution with the use of these agents in the FALD must be taken, as they may precipitate hepatorenal syndrome [11]. Management of severe FALD (patients with cirrhosis or stage III/IV fibrosis) is based upon optimization of the Fontan circulation and hepatology consultation. Supportive care, such as serial paracenteses, can relieve symptoms. In some cases, heart-only transplant may retard worsening of the liver disease; in more advanced cases combined heart-liver transplant may be necessary [8].

\section{Chronic Venous Insufficiency}

Chronic venous insufficiency is common in the Fontan population. Patients with venous insufficiency present with lower extremity edema, venous varicosities, skin changes, and venous ulcers. Valente et al. evaluated the risk of chronic venous insufficiency in a multicenter study of 159 adult Fontan survivors and showed that chronic venous insufficiency was much more common than in healthy controls (60\% versus $32 \%$ ), as was severe venous insufficiency $(22 \%$ versus $0 \%)$. Risk factors for severe chronic venous insufficiency included recurrent groin access for heart catheterization, itching of lower extremities, and a history of deep venous thrombosis [127]. 


\section{Renal Disease}

Patients after Fontan are at risk of kidney injury and often have lower glomerular filtration rate (GFR) than healthy controls. This may reflect end-organ dysfunction caused by low $\mathrm{CO}$ and chronic venous congestion [128]. Younger age at initial Fontan, higher preoperative saturation, and higher preoperative blood pressure independently correlated with higher GFR at latest follow-up. Higher preoperative serum creatinine levels identified patients at highest risk for longterm dysfunction [129]. Opotowsky et al. showed that traditional means of evaluating GFR using creatinine overestimates true GFR and that either cystatin C or urinary biomarkers may be a better reflection of true GFR [130].

\section{Plastic Bronchitis}

Plastic bronchitis is a rare condition after Fontan palliation in which mucofibrinous bronchial casts develop and result in marked airway obstruction. Patients often expectorate large casts or require urgent removal by bronchoscopy, and life-threatening events may occur in up to $40 \%$ of affected patients [131]. Plastic bronchitis occurs in 3-4\% of Fontan patients [132] and is associated with similar risk factors as PLE [133]. Initial management of these patients is similar to that of PLE, including optimization of Fontan hemodynamics and ensuring there is no alternate reversible cause of elevated CVP. Proposed treatment options include inhaled or systemic steroids, aerosolized mucolytics, and aerosolized fibrinolytics such as tissue plasminogen activator. Bronchoscopy is often needed for acute airway rescue [134]. Intervention on abnormal lymphatics has resulted in clinical improvement in isolated patients with Fontan-related plastic bronchitis [135]. OHT can be effective as a long-term option for treating plastic bronchitis [136], although plastic bronchitis has occurred after OHT [137], and plastic bronchitis is a risk-factor for short-term mortality following OHT [138].

\section{Lung Disease}


Patients with Fontan often have a restrictive pattern on pulmonary function testing associated with reduced exercise capacity [139], and this is likely related to multiple prior sternotomies and chest wall deformities such as scoliosis that are very common, particularly following prior thoracotomy [140, 141]. Pulmonary function testing demonstrates reduced forced vital capacity, breathing reserve, and diffusing capacity; and cardiopulmonary exercise testing shows reduced functional capacity [140].

\section{Pregnancy in the Fontan Patient}

Patients with prior Fontan procedure who are contemplating pregnancy should undergo preconception assessment and counseling by an ACHD specialist, including a comprehensive cardiovascular evaluation to identify residual issues following Fontan operation that may adversely affect pregnancy outcome [142]. Women after Fontan are generally fertile, unless they are severely cyanotic. Pregnancy is often well-tolerated in patients with good hemodynamics, reasonably normal oxygen saturation, no atrial arrhythmias, and no history of TE events, but there is still an elevated risk of adverse outcomes compared to healthy controls [142]. Women who have already had significant post-Fontan events or who are likely to be at high risk for such events should be discouraged from attempting pregnancy and should be offered safe and effective forms of contraception. Fontan physiology is a thrombogenic state, thus high-estrogen containing medications are not favored [70]; and instead other forms of contraception should be considered such as intrauterine devices or intramuscular depot progesterone injections.

The largest study to date evaluating pregnancy outcomes after Fontan is now over 20 years old [143] and thus included mostly AP Fontans. This data therefore needs to be interpreted with caution, given that AP Fontans have a much higher overall mortality rate than TCPC [2]. This survey-based study evaluated 116 female patients at several United States centers and 
reported on 33 pregnancies in 14 women. There were 15 (45\%) live births, 13 spontaneous abortions, and 5 elective abortions. There were no maternal complications reported during labor, delivery, or immediate postpartum period [143]. Sporadic cases of women with Fontan palliation having multiple pregnancies and healthy children have also been reported [144, 145] .

Classification schemes have been devised to assess maternal cardiac risk. The World Health Organization (WHO) classification system considers patients after Fontan to be in WHO Pregnancy Risk Category III, the second-highest risk category [146]. Genetic counseling should also be offered, ideally before conception [147]. A monitoring period of 24 hours (including telemetry) with close attention to signs of volume overload or arrhythmia prior to conception also seems reasonable [142]. Premature delivery may be necessary to alleviate maternal symptoms of volume overload or atrial arrhythmia. The most common maternal complications reported during pregnancy in Fontan patients are atrial arrhythmias (especially in those women with atrial arrhythmias prior to pregnancy), TE events, and HF [142, 143].

It is currently uncertain whether and what type of anticoagulation should be used during pregnancy. In general, low-molecular weight heparin is advised for women with a history of a TE event and/or atrial arrhythmia. Vitamin $\mathrm{K}$ antagonists are teratogenic, although they can be used during the second and most of the third trimester of pregnancy with plans to switch to either intravenous heparin infusion or low-molecular weight heparin prior to delivery. In the absence of prior problems most women are treated with just low-dose aspirin, as this had not demonstrated any increase in maternal bleeding events or fetal defects [142].

Regular cardiology follow-up visits during pregnancy, more frequently if the patient becomes symptomatic, are recommended. An interdisciplinary conversation between cardiology, high risk obstetrics, and other clinical staff should be undertaken to determine a detailed delivery 
plan, with plans determined by the start of the third trimester, in the event of a preterm delivery. If the patient becomes symptomatic after the $38^{\text {th }}$ week, labor should be induced, and may need to be induced earlier than that depending on maternal health. There is no clear cardiac indication for Caesarean section in Fontan patients, but it may be necessary for obstetric reasons [142].

\section{SUMMARY}

Despite careful long-term follow-up, there remains continued late attrition of Fontan patients from long-term complications. As surgical techniques for the Fontan operation have improved, overall survival and quality of life have also improved, though Fontan patients remain vulnerable to many cardiac and non-cardiac complications. As this population continues to age, other unexpected comorbidities will likely continue to arise. Management of these patients includes a detailed assessment to treat any correctable lesions, either surgically or with percutaneous techniques. The expert clinician needs to be aware of all potential complications and how to manage them. Management of heart failure and arrhythmias and screening for liver disease are paramount. PLE and plastic bronchitis are also severe, but fortunately rare, complications that require very specialized care and often consideration for transplantation.

Newer testing modalities, such as invasive ambulatory hemodynamic monitoring [148] and newer therapeutics, such as lymphatic interventions, [102, 135] negative pressure ventilation, [149] and emerging blood pump technologies, [58, 150] are also being examined. The role of heart transplant or combined heart-liver transplantation remains controversial. Long-term outcomes for adult patients with Fontan palliation are continually improving, but much work remains to be done to further improve survival and quality of life for this complex cohort of patients. 


\section{References:}

1. Fontan, F., et al., ["Correction" of tricuspid atresia. 2 cases "corrected" using a new surgical technic]. Ann Chir Thorac Cardiovasc, 1971. 10(1): p. 39-47.

2. Pundi, K.N., et al., 40-Year Follow-Up After the Fontan Operation: Long-Term Outcomes of 1,052 Patients. J Am Coll Cardiol, 2015. 66(15): p. 1700-10.

3. Lastinger, L. and A.N. Zaidi, The adult with a fontan: a panacea without a cure? Review of long-term complications. Circ J, 2013. 77(11): p. 2672-81.

4. Khairy, P., et al., Long-term survival, modes of death, and predictors of mortality in patients with Fontan surgery. Circulation, 2008. 117(1): p. 85-92.

5. Cetta, F., et al., Improved early morbidity and mortality after Fontan operation: the Mayo Clinic experience, 1987 to 1992. J Am Coll Cardiol, 1996. 28(2): p. 480-6.

6. Driscoll, D.J., et al., Five- to fifteen-year follow-up after Fontan operation. Circulation, 1992. 85(2): p. 469-96.

7. Deal, B.J. and M.L. Jacobs, Management of the failing Fontan circulation. Heart, 2012. 98(14): p. 1098-104.

8. Daniels, C.J., et al., Fontan-Associated Liver Disease: Proceedings from the American College of Cardiology Stakeholders Meeting, October 1 to 2, 2015, Washington DC. J Am Coll Cardiol, 2017. 70(25): p. 3173-3194.

9. Pundi, K., et al., Liver Disease in Patients After the Fontan Operation. Am J Cardiol, 2016. 117(3): p. 456-60.

10. Ofei, S.Y., et al., Liver fibrosis in adults with Fontan palliation: Do common screening studies predict disease severity? Int J Cardiol, 2015. 181: p. 174-5.

11. Bradley, E., B. Hendrickson, and C. Daniels, Fontan Liver Disease: Review of an Emerging Epidemic and Management Options. Curr Treat Options Cardiovasc Med, 2015. 17(11): p. 51.

12. Kreutzer, G., et al., An operation for the correction of tricuspid atresia. J Thorac Cardiovasc Surg, 1973. 66(4): p. 613-21.

13. Kanakis, M.A., A.C. Petropoulos, and F.A. Mitropoulos, Fontan operation. Hellenic J Cardiol, 2009. 50(2): p. 133-41.

14. Gewillig, M., The Fontan circulation. Heart, 2005. 91(6): p. 839-46.

15. de Leval, M.R., et al., Total cavopulmonary connection: a logical alternative to atriopulmonary connection for complex Fontan operations. Experimental studies and early clinical experience. J Thorac Cardiovasc Surg, 1988. 96(5): p. 682-95.

16. Khairy, P. and N. Poirier, Is the extracardiac conduit the preferred Fontan approach for patients with univentricular hearts? The extracardiac conduit is not the preferred Fontan approach for patients with univentricular hearts. Circulation, 2012. 126(21): p. 2516-25; discussion 2525.

17. Brown, J.W., et al., Lateral tunnel Fontan in the current era: is it still a good option? Ann Thorac Surg, 2010. 89(2): p. 556-62; discussion 562-3.

18. Smas-Suska, M., et al., Long-Term Effects of Percutaneous Fenestration Following the Fontan Procedure in Adult Patients with Congenital Univentricular Heart. Med Sci Monit, 2018. 24: p. 3506-3513.

19. d'Udekem, Y., et al., Redefining expectations of long-term survival after the Fontan procedure: twenty-five years of follow-up from the entire population of Australia and New Zealand. Circulation, 2014. 130(11 Suppl 1): p. S32-8.

20. Schwartz, I., et al., Late outcomes after the Fontan procedure in patients with single ventricle: a meta-analysis. Heart, 2018.

21. Downing, T.E., et al., Long-term survival after the Fontan operation: Twenty years of experience at a single center. J Thorac Cardiovasc Surg, 2017. 154(1): p. 243-253 e2.

22. Dennis, M., et al., Clinical Outcomes in Adolescents and Adults After the Fontan Procedure. J Am Coll Cardiol, 2018. 71(9): p. 1009-1017. 
23. Yabrodi, M. and C.W. Mastropietro, Hypoplastic left heart syndrome: from comfort care to long-term survival. Pediatr Res, 2017. 81(1-2): p. 142-149.

24. Feinstein, J.A., et al., Hypoplastic left heart syndrome: current considerations and expectations. J Am Coll Cardiol, 2012. 59(1 Suppl): p. S1-42.

25. Hirsch, J.C., et al., The lateral tunnel Fontan procedure for hypoplastic left heart syndrome: results of 100 consecutive patients. Pediatr Cardiol, 2007. 28(6): p. 426-32.

26. Salazar, J.D., et al., Fenestration during Fontan palliation: now the exception instead of the rule. J Thorac Cardiovasc Surg, 2010. 140(1): p. 129-36.

27. Wilson, W.M., et al., Outcomes of Patients With Hypoplastic Left Heart Syndrome Reaching Adulthood After Fontan Palliation: Multicenter Study. Circulation, 2018. 137(9): p. 978-981.

28. Texter, K., et al., Building a comprehensive team for the longitudinal care of single ventricle heart defects: Building blocks and initial results. Congenit Heart Dis, 2017. 12(4): p. 403-410.

29. Book, W.M., et al., Clinical Phenotypes of Fontan Failure: Implications for Management. Congenit Heart Dis, 2016. 11(4): p. 296-308.

30. Hebson, C.L., et al., Hemodynamic phenotype of the failing Fontan in an adult population. Am J Cardiol, 2013. 112(12): p. 1943-7.

31. Griffiths, E.R., et al., Evaluating failing Fontans for heart transplantation: predictors of death. Ann Thorac Surg, 2009. 88(2): p. 558-63; discussion 563-4.

32. Shafer, K.M., et al., The importance of the muscle and ventilatory blood pumps during exercise in patients without a subpulmonary ventricle (Fontan operation). J Am Coll Cardiol, 2012. 60(20): p. 2115-21.

33. Goldberg, D.J., et al., Exercise capacity in the Fontan circulation. Cardiol Young, 2013. 23(6): p. 824-30.

34. Cordina, R., et al., Skeletal muscle abnormalities and exercise capacity in adults with a Fontan circulation. Heart, 2013. 99(20): p. 1530-4.

35. Klimes, K., et al., Exercise capacity reflects ventricular function in patients having the Fontan circulation. Cardiol Young, 2009. 19(4): p. 340-5.

36. Peterson, R.J., et al., Noninvasive determination of exercise cardiac function following Fontan operation. J Thorac Cardiovasc Surg, 1984. 88(2): p. 263-72.

37. Baggen, V.J.M., et al., Prognostic Value of Serial N-Terminal Pro-B-Type Natriuretic Peptide Measurements in Adults With Congenital Heart Disease. J Am Heart Assoc, 2018. 7(7).

38. Burchill, L.J., et al., Renin-angiotensin-aldosterone system genotype and serum BNP in a contemporary cohort of adults late after Fontan palliation. Int J Cardiol, 2015. 197: p. 209-15.

39. Eindhoven, J.A., et al., The usefulness of brain natriuretic peptide in complex congenital heart disease: a systematic review. J Am Coll Cardiol, 2012. 60(21): p. 2140-9.

40. Dimopoulos, K., et al., Hyponatraemia: A strong predictor of mortality in adults with congenital heart disease. Eur Heart J, 2010. 31(5): p. 595-601.

41. Opotowsky, A.R., et al., Galectin-3 Is Elevated and Associated With Adverse Outcomes in Patients With Single-Ventricle Fontan Circulation. J Am Heart Assoc, 2016. 5(1).

42. Oldenburger, N.J., et al., Drug therapy in the prevention of failure of the Fontan circulation: a systematic review. Cardiol Young, 2016. 26(5): p. 842-50.

43. Gnanappa, G.K., et al., The Long-Term Management of Children and Adults with a Fontan Circulation: A Systematic Review and Survey of Current Practice in Australia and New Zealand. Pediatr Cardiol, 2017. 38(1): p. 56-69.

44. Wilson, T.G., et al., Use of ACE inhibitors in Fontan: Rational or irrational? Int J Cardiol, 2016. 210: p. 95-9. 
45. Cobden, I., et al., Captopril in the hepatorenal syndrome. J Clin Gastroenterol, 1985. 7(4): p. 354-60.

46. Kouatli, A.A., et al., Enalapril does not enhance exercise capacity in patients after Fontan procedure. Circulation, 1997. 96(5): p. 1507-12.

47. Shaddy, R.E., et al., Carvedilol for children and adolescents with heart failure: a randomized controlled trial. JAMA, 2007. 298(10): p. 1171-9.

48. Ishibashi, N., et al., Effect of carvedilol on heart failure in patients with a functionally univentricular heart. Circ J, 2011. 75(6): p. 1394-9.

49. Ishibashi, N., et al., Effectiveness of carvedilol for congestive heart failure that developed long after modified Fontan operation. Pediatr Cardiol, 2006. 27(4): p. 473-5.

50. Ishibashi, T., et al., Effect of carvedilol on venous return: a mechanism of reduction in blood pressure. Jpn J Pharmacol, 1991. 55(1): p. 186-9.

51. Giardini, A., et al., A pilot study on the effects of carvedilol on right ventricular remodelling and exercise tolerance in patients with systemic right ventricle. Int $\mathrm{J}$ Cardiol, 2007. 114(2): p. 241-6.

52. McMurray, J.J., et al., Dual angiotensin receptor and neprilysin inhibition as an alternative to angiotensin-converting enzyme inhibition in patients with chronic systolic heart failure: rationale for and design of the Prospective comparison of ARNI with ACEI to Determine Impact on Global Mortality and morbidity in Heart Failure trial (PARADIGMHF). Eur J Heart Fail, 2013. 15(9): p. 1062-73.

53. Hebert, A., et al., Bosentan improves exercise capacity in adolescents and adults after Fontan operation: the TEMPO (Treatment With Endothelin Receptor Antagonist in Fontan Patients, a Randomized, Placebo-Controlled, Double-Blind Study Measuring Peak Oxygen Consumption) study. Circulation, 2014. 130(23): p. 2021-30.

54. Schuuring, M.J., et al., Impact of bosentan on exercise capacity in adults after the Fontan procedure: a randomized controlled trial. Eur J Heart Fail, 2013. 15(6): p. 690-8.

55. Cedars, A.M., et al., Effect of Ambrisentan on Exercise Capacity in Adult Patients After the Fontan Procedure. Am J Cardiol, 2016. 117(9): p. 1524-32.

56. Demetriades, P., et al., The use of Macitentan in Fontan circulation: a case report. BMC Cardiovasc Disord, 2017. 17(1): p. 131.

57. Buratto, E., et al., Ventricular assist devices for the failing univentricular circulation. Expert Rev Med Devices, 2017. 14(6): p. 449-459.

58. Rodefeld, M.D., S.H. Frankel, and G.A. Giridharan, Cavopulmonary assist: (em)powering the univentricular fontan circulation. Semin Thorac Cardiovasc Surg Pediatr Card Surg Annu, 2011. 14(1): p. 45-54.

59. Cunningham, J.W., et al., Decline in peak oxygen consumption over time predicts death or transplantation in adults with a Fontan circulation. Am Heart J, 2017. 189: p. 184-192.

60. Bryant, R., 3rd and D. Morales, Overview of adult congenital heart transplants. Ann Cardiothorac Surg, 2018. 7(1): p. 143-151.

61. Alshawabkeh, L.I. and A.R. Opotowsky, Burden of Heart Failure in Adults with Congenital Heart Disease. Curr Heart Fail Rep, 2016. 13(5): p. 247-254.

62. Alshawabkeh, L.I., et al., Wait-List Outcomes for Adults With Congenital Heart Disease Listed for Heart Transplantation in the U.S. J Am Coll Cardiol, 2016. 68(9): p. 908-17.

63. D'Souza, B.A., et al., Single-center outcomes of combined heart and liver transplantation in the failing Fontan. Clin Transplant, 2017. 31(3).

64. Faircloth, J.M., et al., Intermediate term thrombotic risk in contemporary total cavopulmonary connection for single ventricle circulations. J Thromb Thrombolysis, 2017. 44(3): p. 275-280.

65. Alsaied, T., et al., Strategies for thromboprophylaxis in Fontan circulation: a metaanalysis. Heart, 2015. 101(21): p. 1731-7. 
66. Takeuchi, D., et al., Blood coagulation abnormalities and the usefulness of D-dimer level for detecting intracardiac thrombosis in adult Fontan patients. Int J Cardiol, 2016. 224: p. 139-144.

67. Egbe, A.C., et al., Thrombotic and Embolic Complications Associated With Atrial Arrhythmia After Fontan Operation: Role of Prophylactic Therapy. J Am Coll Cardiol, 2016. 68(12): p. 1312-9.

68. Khairy, P., et al., Thromboprophylaxis for atrial arrhythmias in congenital heart disease: A multicenter study. Int J Cardiol, 2016. 223: p. 729-735.

69. Faircloth, J.M., et al., Time in therapeutic range as a marker for thrombotic and bleeding outcomes in Fontan patients. J Thromb Thrombolysis, 2017. 44(1): p. 38-47.

70. Warnes, C.A., et al., ACC/AHA 2008 Guidelines for the Management of Adults with Congenital Heart Disease: a report of the American College of Cardiology/American Heart Association Task Force on Practice Guidelines (writing committee to develop guidelines on the management of adults with congenital heart disease). Circulation, 2008. 118(23): p. e714-833.

71. Yang, H., B.J. Bouma, and B.J.M. Mulder, Is Initiating NOACs for Atrial Arrhythmias Safe in Adults with Congenital Heart Disease? Cardiovasc Drugs Ther, 2017. 31(4): p. 413417.

72. Georgekutty, J., et al., Novel oral anticoagulant use in adult Fontan patients: $A$ single center experience. Congenit Heart Dis, 2018.

73. Wan, D., et al., Anticoagulation for Thromboembolic Risk Reduction in Adults With Congenital Heart Disease. Can J Cardiol, 2017. 33(12): p. 1597-1603.

74. Walsh, E.P. and F. Cecchin, Arrhythmias in adult patients with congenital heart disease. Circulation, 2007. 115(4): p. 534-45.

75. Durongpisitkul, K., et al., Predictors of early- and late-onset supraventricular tachyarrhythmias after Fontan operation. Circulation, 1998. 98(11): p. 1099-107.

76. Gelatt, M., et al., Risk factors for atrial tachyarrhythmias after the Fontan operation. J Am Coll Cardiol, 1994. 24(7): p. 1735-41.

77. Labombarda, F., et al., Acute amiodarone-induced pulmonary toxicity: an association of risk factors in a child operated by arterial switch operation. Congenit Heart Dis, 2008. 3(5): p. 365-7.

78. Haas, N.A. and C.K. Camphausen, Impact of early and standardized treatment with amiodarone on therapeutic success and outcome in pediatric patients with postoperative tachyarrhythmia. J Thorac Cardiovasc Surg, 2008. 136(5): p. 1215-22.

79. Oski, J.A., et al., Embolic stroke after ligation of the pulmonary artery in patients with functional single ventricle. Am Heart J, 1996. 132(4): p. 836-40.

80. Nehgme, R.A., et al., Transthoracic percutaneous access for electroanatomic mapping and catheter ablation of atrial tachycardia in patients with a lateral tunnel Fontan. Heart Rhythm, 2006. 3(1): p. 37-43.

81. Aoki, $\mathrm{H}$., et al., A new procedure for a trans-conduit puncture by grasping the dilator tip with a snare catheter: an alternative access method during catheter ablation of supraventricular tachycardias after an extracardiac Fontan operation. Heart Rhythm, 2014. 11(8): p. 1492-4.

82. Soejima, K., et al., Subxiphoid surgical approach for epicardial catheter-based mapping and ablation in patients with prior cardiac surgery or difficult pericardial access.

Circulation, 2004. 110(10): p. 1197-201.

83. Mavroudis, C., et al., Fontan conversion to cavopulmonary connection and arrhythmia circuit cryoblation. J Thorac Cardiovasc Surg, 1998. 115(3): p. 547-56.

84. Deal, B.J., et al., Impact of arrhythmia circuit cryoablation during Fontan conversion for refractory atrial tachycardia. Am J Cardiol, 1999. 83(4): p. 563-8. 
85. Mavroudis, C., et al., The favorable impact of arrhythmia surgery on total cavopulmonary artery Fontan conversion. Semin Thorac Cardiovasc Surg Pediatr Card Surg Annu, 1999. 2: p. 143-156.

86. Mavroudis, C., et al., Total cavopulmonary conversion and maze procedure for patients with failure of the Fontan operation. J Thorac Cardiovasc Surg, 2001. 122(5): p. 863-71.

87. Rostock, T., et al., Radiofrequency catheter ablation of a macroreentrant ventricular tachycardia late after surgical repair of tetralogy of Fallot using the electroanatomic mapping (CARTO). Pacing Clin Electrophysiol, 2004. 27(6 Pt 1): p. 801-4.

88. Hauck, A., et al., The Pulmonary Circulation in the Single Ventricle Patient. Children (Basel), 2017. 4(8).

89. Pereira-da-Silva, T., et al., Percutaneous occlusion of vascular malformations in pediatric and adult patients: 20-year experience of a single center. Catheter Cardiovasc Interv, 2016. 87(2): p. E62-8.

90. Poterucha, J.T., et al., Embolization of Veno-venous Collaterals after the Fontan Operation Is Associated with Decreased Survival. Congenit Heart Dis, 2015. 10(5): p. E230-6.

91. Cohen, M.I., et al., Atrial pacing: an alternative treatment for protein-losing enteropathy after the Fontan operation. J Thorac Cardiovasc Surg, 2001. 121(3): p. 582-3.

92. Veldtman, G.R. and G.D. Webb, Improved survival in Fontan-associated protein-losing enteropathy. J Am Coll Cardiol, 2014. 64(1): p. 63-5.

93. John, A.S., et al., Clinical outcomes and improved survival in patients with protein-losing enteropathy after the Fontan operation. J Am Coll Cardiol, 2014. 64(1): p. 54-62.

94. Johnson, J.N., D.J. Driscoll, and P.W. O'Leary, Protein-losing enteropathy and the Fontan operation. Nutr Clin Pract, 2012. 27(3): p. 375-84.

95. Rychik, J., The Relentless Effects of the Fontan Paradox. Semin Thorac Cardiovasc Surg Pediatr Card Surg Annu, 2016. 19(1): p. 37-43.

96. Rychik, J., Protein-losing enteropathy after Fontan operation. Congenit Heart Dis, 2007. 2(5): p. 288-300.

97. Udink Ten Cate, F.E., et al., Towards a proposal for a universal diagnostic definition of protein-losing enteropathy in Fontan patients: a systematic review. Heart, 2016. 102(14): p. 1115-9.

98. Meadows, J., K. Gauvreau, and K. Jenkins, Lymphatic obstruction and protein-losing enteropathy in patients with congenital heart disease. Congenit Heart Dis, 2008. 3(4): p. 269-76.

99. Menon, S., et al., Role of caval venous manipulation in treatment of protein-losing enteropathy. Cardiol Young, 2008. 18(3): p. 275-81.

100. Vyas, H., et al., Results of transcatheter Fontan fenestration to treat protein losing enteropathy. Catheter Cardiovasc Interv, 2007. 69(4): p. 584-9.

101. Thacker, D., et al., Use of oral budesonide in the management of protein-losing enteropathy after the Fontan operation. Ann Thorac Surg, 2010. 89(3): p. 837-42.

102. Dori, Y., Novel Lymphatic Imaging Techniques. Tech Vasc Interv Radiol, 2016. 19(4): p. 255-261.

103. Guez, D., et al., Transhepatic lymphatic embolization of intractable hepatic lymphorrhea. J Vasc Interv Radiol, 2014. 25(1): p. 149-50.

104. Itkin, M., et al., Protein-Losing Enteropathy in Patients With Congenital Heart Disease. J Am Coll Cardiol, 2017. 69(24): p. 2929-2937.

105. Lewis, M.J., et al., Serial cardiac MRIs in adult Fontan patients detect progressive hepatic enlargement and congestion. Congenit Heart Dis, 2017. 12(2): p. 153-158.

106. Ghaferi, A.A. and G.M. Hutchins, Progression of liver pathology in patients undergoing the Fontan procedure: Chronic passive congestion, cardiac cirrhosis, hepatic adenoma, and hepatocellular carcinoma. J Thorac Cardiovasc Surg, 2005. 129(6): p. 1348-52. 
107. Camposilvan, S., et al., Liver and cardiac function in the long term after Fontan operation. Ann Thorac Surg, 2008. 86(1): p. 177-82.

108. Kirklin, J.K., et al., Challenges of Cardiac Transplantation Following the Fontan Procedure. World J Pediatr Congenit Heart Surg, 2017. 8(4): p. 480-486.

109. Elder, R.W., et al., Features of portal hypertension are associated with major adverse events in Fontan patients: the VAST study. Int J Cardiol, 2013. 168(4): p. 3764-9.

110. Assenza, G.E., et al., MELD-XI score and cardiac mortality or transplantation in patients after Fontan surgery. Heart, 2013. 99(7): p. 491-6.

111. Evans, W.N., et al., MELD-XI Scores Correlate with Post-Fontan Hepatic Biopsy Fibrosis Scores. Pediatr Cardiol, 2016. 37(7): p. 1274-7.

112. Sheth, M., M. Riggs, and T. Patel, Utility of the Mayo End-Stage Liver Disease (MELD) score in assessing prognosis of patients with alcoholic hepatitis. BMC Gastroenterol, 2002. 2: p. 2.

113. Surrey, L.F., et al., Prevalence and characterization of fibrosis in surveillance liver biopsies of patients with Fontan circulation. Hum Pathol, 2016. 57: p. 106-115.

114. Wu, F.M., et al., Portal and centrilobular hepatic fibrosis in Fontan circulation and clinical outcomes. J Heart Lung Transplant, 2015. 34(7): p. 883-91.

115. Schwartz, M.C., et al., Portal and sinusoidal fibrosis are common on liver biopsy after Fontan surgery. Pediatr Cardiol, 2013. 34(1): p. 135-42.

116. Thielmann, M., et al., Risk prediction and outcomes in patients with liver cirrhosis undergoing open-heart surgery. Eur J Cardiothorac Surg, 2010. 38(5): p. 592-9.

117. Wallihan, D.B. and D.J. Podberesky, Hepatic pathology after Fontan palliation: spectrum of imaging findings. Pediatr Radiol, 2013. 43(3): p. 330-8.

118. Wu, F.M., et al., Liver disease in the patient with Fontan circulation. Congenit Heart Dis, 2011. 6(3): p. 190-201.

119. Malinchoc, M., et al., A model to predict poor survival in patients undergoing transjugular intrahepatic portosystemic shunts. Hepatology, 2000. 31(4): p. 864-71.

120. Ginde, S., et al., Noninvasive assessment of liver fibrosis in adult patients following the Fontan procedure. Congenit Heart Dis, 2012. 7(3): p. 235-42.

121. Millonig, G., et al., Liver stiffness is directly influenced by central venous pressure. $\mathrm{J}$ Hepatol, 2010. 52(2): p. 206-10.

122. Kuwabara, M., et al., Liver Cirrhosis and/or Hepatocellular Carcinoma Occurring Late After the Fontan Procedure- A Nationwide Survey in Japan. Circ J, 2018. 82(4): p. 11551160.

123. Poterucha, J.T., et al., Magnetic Resonance Elastography: A Novel Technique for the Detection of Hepatic Fibrosis and Hepatocellular Carcinoma After the Fontan Operation. Mayo Clin Proc, 2015. 90(7): p. 882-94.

124. Nandwana, S.B., et al., Abdominal Imaging Surveillance in Adult Patients After Fontan Procedure: Risk of Chronic Liver Disease and Hepatocellular Carcinoma. Curr Probl Diagn Radiol, 2018. 47(1): p. 19-22.

125. Wells, M.L., et al., Benign nodules in post-Fontan livers can show imaging features considered diagnostic for hepatocellular carcinoma. Abdom Radiol (NY), 2017. 42(11): p. 2623-2631.

126. Grace, J.A., et al., Update on new aspects of the renin-angiotensin system in liver disease: clinical implications and new therapeutic options. Clin Sci (Lond), 2012. 123(4): p. 225-39.

127. Valente, A.M., et al., The CALF (Congenital Heart Disease in Adults Lower Extremity Systemic Venous Health in Fontan Patients) study. J Am Coll Cardiol, 2010. 56(2): p. 144-50.

128. Mori, M., et al., Beyond a broken heart: circulatory dysfunction in the failing Fontan. Pediatr Cardiol, 2014. 35(4): p. 569-79. 
129. Sammour, F., et al., Renal function of patients with a failing Fontan circuit undergoing total cavopulmonary revision surgery. Pediatr Cardiol, 2009. 30(3): p. 282-8.

130. Opotowsky, A.R., et al., Estimated glomerular filtration rate and urine biomarkers in patients with single-ventricle Fontan circulation. Heart, 2017. 103(6): p. 434-442.

131. Brogan, T.V., et al., Plastic bronchitis in children: a case series and review of the medical literature. Pediatr Pulmonol, 2002. 34(6): p. 482-7.

132. Caruthers, R.L., et al., Demographic characteristics and estimated prevalence of Fontanassociated plastic bronchitis. Pediatr Cardiol, 2013. 34(2): p. 256-61.

133. Schumacher, K.R., et al., Risk factors and outcome of Fontan-associated plastic bronchitis: a case-control study. J Am Heart Assoc, 2014. 3(2): p. e000865.

134. Soyer, T., et al., Use of serial rigid bronchoscopy in the treatment of plastic bronchitis in children. J Pediatr Surg, 2016. 51(10): p. 1640-3.

135. Dori, Y., et al., Successful treatment of plastic bronchitis by selective lymphatic embolization in a Fontan patient. Pediatrics, 2014. 134(2): p. e590-5.

136. Gossett, J.G., et al., Outcomes of cardiac transplantation in single-ventricle patients with plastic bronchitis: a multicenter study. J Am Coll Cardiol, 2013. 61(9): p. 985-6.

137. Parent, J.J. and R.K. Darragh, Severe airway obstruction from a bronchial cast after cardiac transplantation. Cardiol Young, 2014. 24(4): p. 739-40.

138. Parent, J.J., et al., Strategies to Prevent Cast Formation in Patients with Plastic Bronchitis Undergoing Heart Transplantation. Pediatr Cardiol, 2017. 38(5): p. 10771079.

139. Cohen, S.B., et al., Extracardiac complications in adults with congenital heart disease. Congenit Heart Dis, 2013. 8(5): p. 370-80.

140. Opotowsky, A.R., et al., Abnormal spirometry after the Fontan procedure is common and associated with impaired aerobic capacity. Am J Physiol Heart Circ Physiol, 2014. 307(1): p. H110-7.

141. Fredriksen, P.M., et al., Lung function and aerobic capacity in adult patients following modified Fontan procedure. Heart, 2001. 85(3): p. 295-9.

142. Canobbio, M.M., et al., Management of Pregnancy in Patients With Complex Congenital Heart Disease: A Scientific Statement for Healthcare Professionals From the American Heart Association. Circulation, 2017. 135(8): p. e50-e87.

143. Canobbio, M.M., et al., Pregnancy outcomes after the Fontan repair. J Am Coll Cardiol, 1996. 28(3): p. 763-7.

144. Al Najashi, K.S., et al., Four successful pregnancies in a patient with Fontan palliation and congenital heart disease: a case report. J Cardiothorac Surg, 2017. 12(1): p. 104.

145. Opotowsky, A.R., et al., Successful pregnancies in two women with hypoplastic left heart syndrome. Congenit Heart Dis, 2010. 5(5): p. 476-81.

146. Thorne, S., A. MacGregor, and C. Nelson-Piercy, Risks of contraception and pregnancy in heart disease. Heart, 2006. 92(10): p. 1520-5.

147. Morales, A., et al., At the Heart of the Pregnancy: What Prenatal and Cardiovascular Genetic Counselors Need to Know about Maternal Heart Disease. J Genet Couns, 2017. 26(4): p. 669-688.

148. Bradley, E.A., D. Berman, and C.J. Daniels, First implantable hemodynamic monitoring device placement in single ventricle fontan anatomy. Catheter Cardiovasc Interv, 2016. 88(2): p. 248-52.

149. Deshpande, S.R. and K.O. Maher, Long term negative pressure ventilation: Rescue for the failing fontan? World J Cardiol, 2014. 6(8): p. 861-4.

150. Jaquiss, R.D. and H. Aziz, Is Four Stage Management the Future of Univentricular Hearts? Destination Therapy in the Young. Semin Thorac Cardiovasc Surg Pediatr Card Surg Annu, 2016. 19(1): p. 50-4. 


\section{Figure Legend}

Figure 1:

Types of Fontan connection: A) atriopulmonary Fontan, with anastomosis of right atrial appendage directly to pulmonary artery; B) lateral caval Fontan, with a patch separating the venous inflow of the right atrium from the right atrial body, and anastomosis of superior vena cava to pulmonary artery; C) extracardiac Fontan, with a prosthetic tube conveying blood completely outside the body of the right atrium to the cavopulmonary anastomosis.

Figure 2:

Three-dimensional CT scan reconstruction of an atriopulmonary Fontan connection in a 30 year old woman scheduled for Fontan conversion to extracardiac Fontan. RA = right atrium; RAA = right atrial appendage; Ao = aorta; PA = pulmonary artery

Figure 3:

Angiogram in a 25 year old woman before and after Fontan conversion from AP Fontan to extracardiac Fontan. (A) Prior to Fontan conversion showing massive dilated right atrium. (B) Injection into IVC after Fontan conversion showing extracardiac tube connecting IVC directly to $\mathrm{PA} . \mathrm{RA}=$ right atrium; IVC = inferior vena cava; $E C=$ extracardiac conduit; $\mathrm{PA}=$ pulmonary artery

Figure 4:

(A) Angiogram of a 28 year old lateral tunnel Fontan patient with exercise-induced cyanosis due to formation of a decompressing venovenous collateral. SVC = superior vena cava; PA = pulmonary artery. Red arrow showing location of venovenous collateral from innominate vein draining to pulmonary veins. (B) Arrow demonstrating site of successful coil embolization of collateral. 


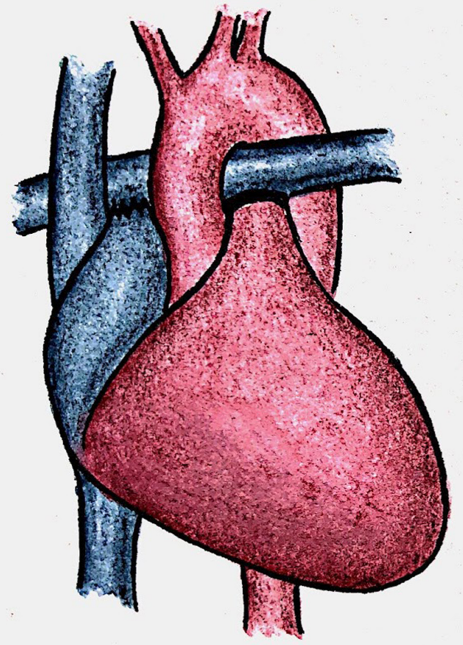

A

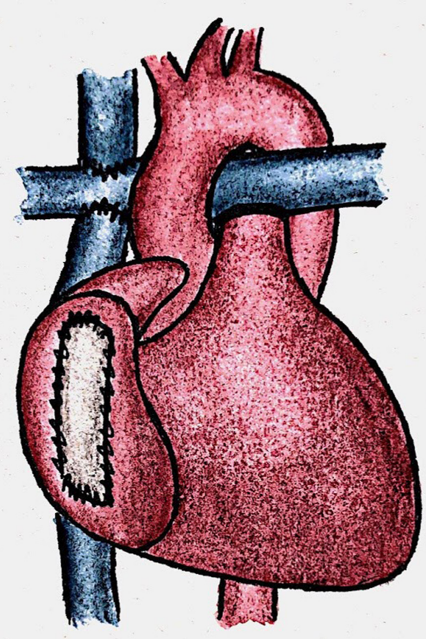

B

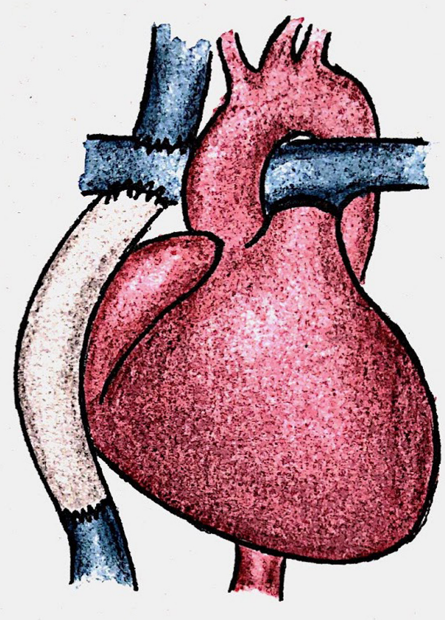

C

Figure 1 




Figure 2 


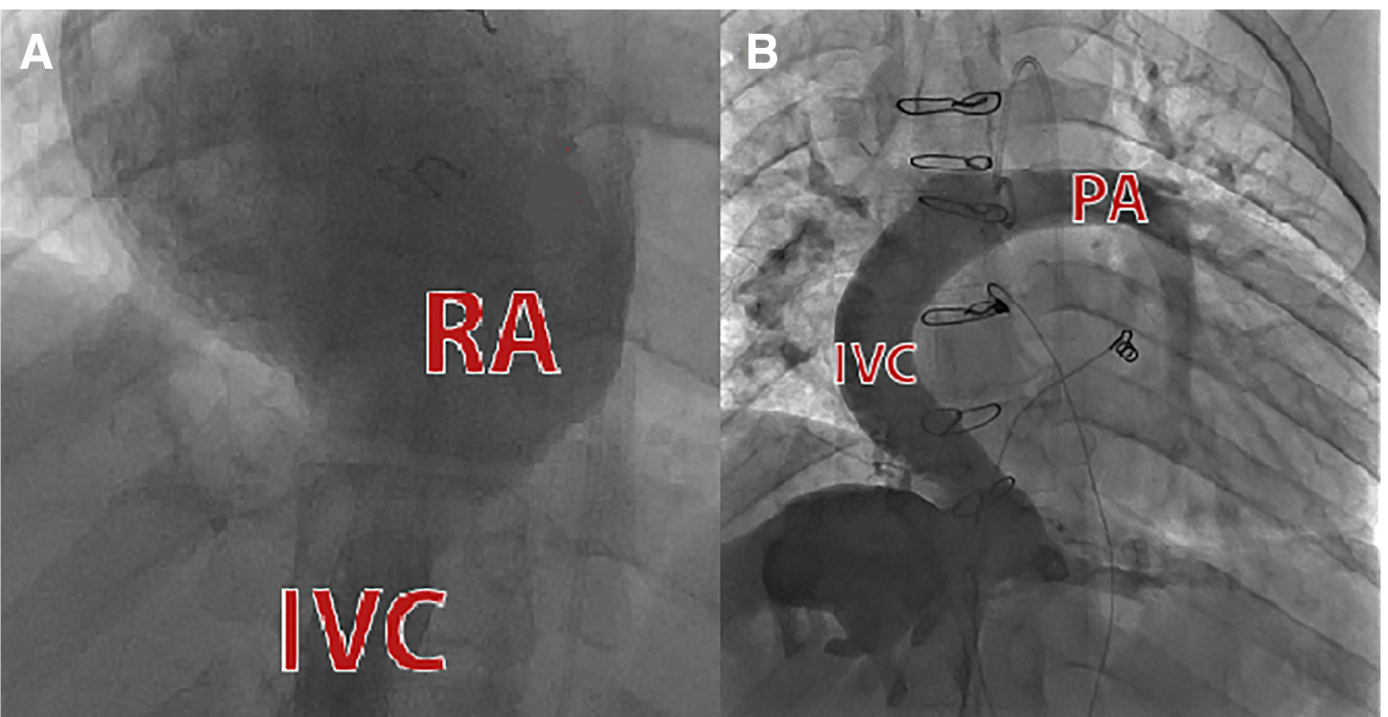

Figure 3 


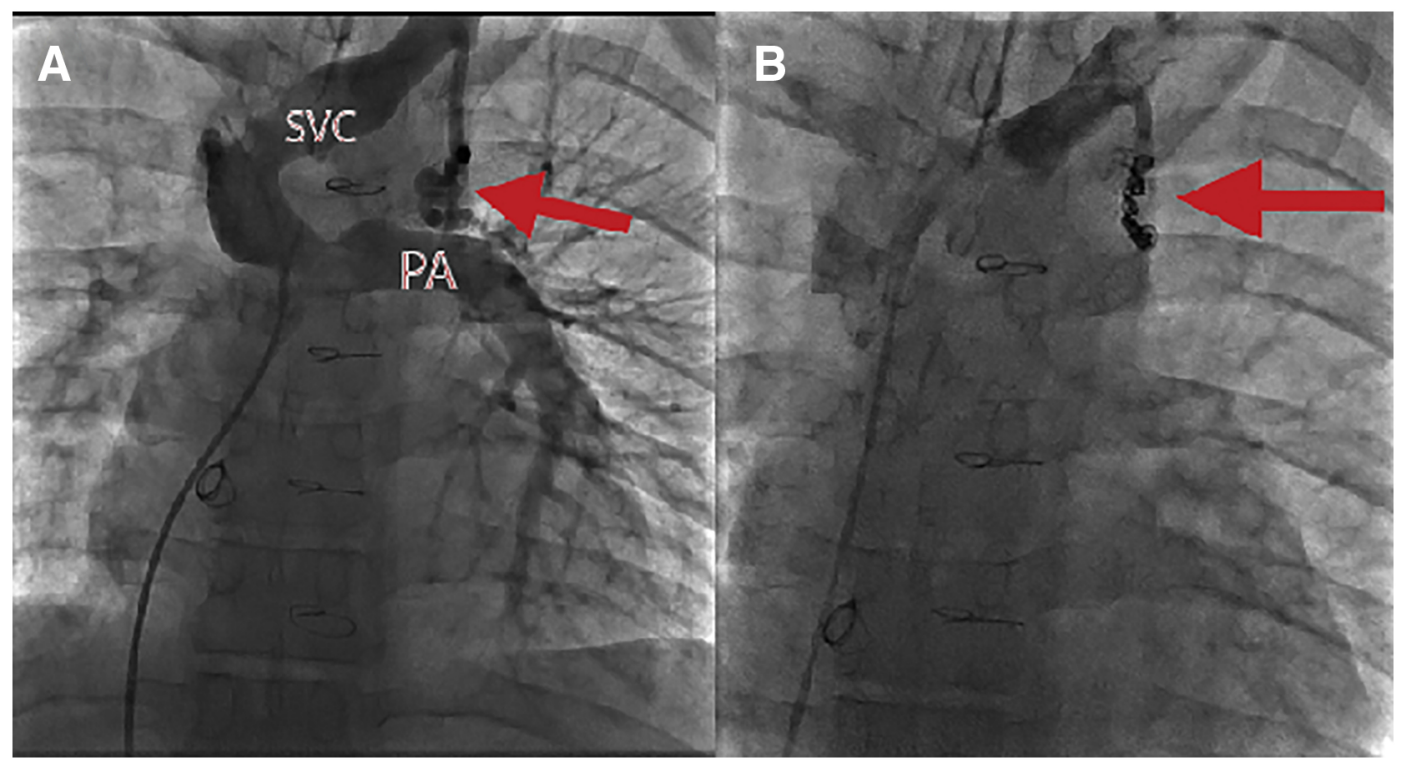

Figure 4 